\title{
Comparison of apparent diffusion coefficient maps to T2-weighted images for target delineation in cervix cancer brachytherapy
}

\author{
Jacqueline Esthappan, PhD', Daniel J. Ma, MD', Vamsidhar R. Narra, MD², Constantine A. Raptis, MD², Perry W. Grigsby, MD' \\ IDepartment of Radiation Oncology, Washington University School of Medicine, St. Lovis, 2Mallinckrodt Institute of Radiology, St. Louis, USA
}

\begin{abstract}
Purpose: T2-weighted (T2W) magnetic resonance imaging (MRI) has been used for target delineation in cervix cancer brachytherapy. The objective of this study was to examine the feasibility of using diffusion-weighted magnetic resonance imaging (DWI) for target delineation as compared against T2W imaging.

Material and methods: Fifteen cervix cancer patients, implanted with tandem and ovoid applicators, underwent T2W turbo-spin echo imaging and DWI with a maximum diffusion factor of $800 \mathrm{sec} / \mathrm{mm}^{2}$ on a 1.5-T MR scanner. Apparent diffusion coefficient (ADC) maps were derived from the DWI. The gross tumor volume was manually delineated on the T2W and ADC datasets for each patient. The agreement between T2W- and ADC-delineated volumes was assessed using the Dice similarity coefficient (DSC). An algorithm was developed to compare the edge contrast of the delineated volumes on T2W images and ADC maps by calculating the percentage difference in the intensity values of selected regions of pixels inside versus outside the target contour.

Results: ADC-delineated volumes were generally smaller than T2W-delineated volumes, yielding a low DSC of 0.54 \pm 0.22 . ADC maps were found to display superior definition of the target volume edge relative to T2W images, yielding a statistically significant difference between the mean edge contrast on ADC $(12.7 \pm 7.7 \%)$ versus that on T2W images $(4.6 \pm 3.2 \% ; p=0.0010)$.

Conclusions: These results suggest that incorporating the use of DWI for cervix cancer brachytherapy may yield gross tumor volumes that are different from those based on T2W images alone.

J Contemp Brachyther 2011; 3, 4: 193-198 DOI: 10.5114/jcb.2011.26470
\end{abstract}

Key words: cervix cancer, brachytherapy, diffusion weighted imaging, target delineation.

\section{Purpose}

The superiority of magnetic resonance imaging (MRI) over computed tomography (CT) and ultrasound has been reported for visualizing cervical tumor size and extent of disease [1]. More recent guidelines for intracavitary brachytherapy planning for cervix cancer recommend the use of T2-weighted (T2W) imaging sequences for target delineation [2-4]. Cervix tumors display slightly higher signal intensities relative to normal cervical tissues on T2W images, aiding in the determination of tumor size, location, and spread to other tissues [2-4]. Recent work suggests that another MR imaging sequence, referred to as diffusion-weighted imaging (DWI), may also be useful for the diagnosis and staging of cervical cancer [5-13]. As of yet there are no published studies looking at the use of DWI for image-guided radiation therapy of cervix cancer. The objective of this study was to examine the feasibility of using DWI for target delineation in cervix cancer brachytherapy as compared against T2W imaging.
DWI is a magnetic resonance imaging technique that is sensitive to the motion of water molecules [7]. The water diffusion properties of different tissues can be quantified on the DWI as an apparent diffusion coefficient (ADC) value. From DW images, ADC values are derived in the following manner:

$$
\mathrm{ADC} \text { value }=\frac{1}{\mathrm{~b}} \ln \frac{\mathrm{So}}{\mathrm{S}_{\mathrm{DW}}}
$$

where $S_{D W}$ and So are signal intensities measured with and without diffusion-weighted gradients, respectively, and the $b$-value is a diffusion factor which is a function of imaging technique [6]. It has been suggested that due to increased cellularity and, hence, reduced mobility of water molecules in tumors, ADC values should be lower in tumors than in benign tissues. A number of studies have demonstrated this difference in ADC value for a number of sites, including brain, breast, and hepatic tumors, as well 
as cervix tumors, and have concluded that measurement of ADC values may provide a quantitative tool for diagnosis and for evaluating therapy response [6-14]. The diffusion properties of tissues in vivo can be concisely presented as a two-dimensional map, where the pixel intensities are proportional to the ADC value. The differences in ADC values may lead to improved soft tissue contrast between tumor and normal tissues on ADC maps relative to $\mathrm{T} 2 \mathrm{~W}$ images. Charles-Edwards et al. found that the accuracy of detecting disease for stage Ia-Ib1 cervix cancer patients improved when using ADC maps in addition to $\mathrm{T} 2 \mathrm{~W}$ images [6]. These results suggest that delineation, in addition to detection, of the tumor for radiotherapy may improve with the addition of ADC mapping.

The purpose of the current study was to evaluate the feasibility of using ADC maps for intracavitary brachytherapy treatment planning for cervix cancer, i.e., to see how this previously reported difference in ADC value between malignant and normal cervix tissues affects target visibility and target volume delineation on ADC maps, as compared to the current standard of T2W images.

\section{Material and methods}

\section{Patients}

Fifteen patients with stage IB1-IIIB cervical cancer were accrued over a ten-month period (May 2008 to February 2009) on a research protocol approved by the Washington University Human Research Protection Office. Informed consent was waived for this retrospective study. Patients were prescribed to receive high dose rate brachytherapy in six weekly fractions concurrently with a 50.4 Gy external beam dose in 28 fractions to the pelvis using intensitymodulated radiation therapy. Brachytherapy dose was based on stage and tumor size at the time of diagnosis.

\section{Imaging}

At the time of brachytherapy, patients were implanted with Fletcher-style titanium tandem and colpostats without shielding, followed by imaging on a 1.5-T MRI scanner (Brilliance 230W, Philips Medical Systems, Cleveland, Ohio ${ }^{\circledR}$ ). Patients were positioned supine in body/ pelvis phased array surface coils. Scanning sequences included T2W turbo-spin echo imaging (repetition time, TR, of $3200 \mathrm{~ms}$ and echo time, TE, of $100 \mathrm{~ms}$, in-plane resolution of $0.2 \mathrm{~cm}$ or less, section thickness $0.5 \mathrm{~cm}$, no intersection gap, axial and parasagittal acquisition planes) and single-shot DW echo-planar imaging (TR $1300 \mathrm{~ms}$, TE $75 \mathrm{~ms}$, in-plane resolution of $0.2 \mathrm{~cm}$ or less, section thickness $0.5 \mathrm{~cm}$, parasagittal acquisition plane only) with $b$-values of 0 and $800 \mathrm{~s} / \mathrm{mm}^{2}$. Diffusion gradients were applied in three directions. Imaging extents were set approximately from L4 to the ischial tuberosities in the craniocaudal direction, from the abdominal wall to the sacrum in the anterior-posterior direction, and were set to include the iliac crests in the transverse direction. Apparent diffusion coefficient (ADC) maps were generated from the diffusion weighted images on the scanner console software.

\section{Target delineations on ADC maps versus T2W images}

T2W images and ADC maps at the time of the first brachytherapy fraction were transferred to a radiation therapy treatment planning system (Eclipse TPS, Varian Medical Systems, Palo Alto, $\mathrm{CA}^{\circledR}$ ) for contouring and isodose planning. Because the T2W and DWI were acquired in the same imaging session, the datasets, upon import into the TPS, were automatically registered based on fixed DICOM coordinates. The registration between image datasets was manually verified by checking the rigid registration of the vertebral bodies, and if necessary, adjusted in the region of interest containing the uterus. The delineation of target volumes on T2W images and ADC maps was carried out according to the definition of gross tumor volume (GTV), i.e., primary tumor in the cervix and any parametrial extension as detected by clinical examination and visualized on MRI [2-4]. All target delineations were done manually on the sagittal acquisitions for the T2W images and also on the ADC maps for each patient by the same observer, an expert physician trained in gynecologic brachytherapy. The agreement between T2W- and ADCdelineated target volumes was assessed using the Dice similarity coefficient (DSC), defined as:

$$
\mathrm{DSC}=\frac{\mathrm{V}_{\mathrm{T} 2 \mathrm{~W}}-\mathrm{ADC}}{\left(\left(\mathrm{V}_{\mathrm{T} 2 \mathrm{~W}}+\mathrm{V}_{\mathrm{ADC}}\right) / 2\right)}
$$

where $V_{T 2 W}$ is the target volume delineated on the T2W images, $V_{A D C}$ is the target volume delineated on the ADC maps, and $V_{T 2 W-A D C}$ is the overlap of the target volumes delineated on T2W images and ADC maps [15].

\section{Comparison of target edge visibility on $\mathrm{T} 2 \mathrm{~W}$ images and ADC maps}

The target volume contours and image datasets were input to an algorithm developed to quantify the intensity of the target edge visible on $\mathrm{T} 2 \mathrm{~W}$ images and ADC maps. The algorithm was used to track the contour on each image slice and calculate the percent difference in the mean intensity value of "inner pixels", i.e., those pixels inside the target contour, versus "outer pixels", i.e., those pixels outside the target contour. Only intensities within a $3 \times 3$ pixel region centered about the contour were used in the calculation. The percentage edge contrast was defined as

$\underset{\text { Edge con- }}{\text { trast }(\%)}=100 \times \frac{[\langle\text { outer pixel values }\rangle-\langle\text { inner pixel values }\rangle]}{\langle\text { outer pixel values }\rangle}$

where the edge contrast was calculated in this manner for the T2W- and ADC-delineated target volumes for each patient, and compared to one another using the Sign test.

\section{Results}

Table 1 shows the $\mathrm{V}_{\mathrm{T} 2 \mathrm{~W}-\mathrm{ADC}}, \mathrm{V}_{\mathrm{T} 2 \mathrm{~W}}$, and $\mathrm{V}_{\mathrm{ADC}}$, for all fifteen patients. For thirteen out of fifteen patients, we 
found that the $\mathrm{V}_{\mathrm{ADC}}$ was less than the $\mathrm{V}_{\mathrm{T} 2 \mathrm{~W}}$. The mean ADC-delineated target volume was less than the T2Wdelineated target volume $\left(52.3 \pm 54.6 \mathrm{~cm}^{3}\right.$ vs. $64.0 \pm$ $70.5 \mathrm{~cm}^{3}, p=0.0074$ Sign test). The DSC values are also reported in Table 1, where a DSC greater than 0.70 indicates excellent agreement [16]. For one patient, Patient 7, the DSC was zero, but the $\mathrm{V}_{\mathrm{T} 2 \mathrm{~W}}$ and $\mathrm{V}_{\mathrm{ADC}}$ were also very small, less than $1.0 \mathrm{~cm}^{3}$ on the ADC maps. Similarly, low DSC values were seen for two other patients, Patients 8 and 12 , where the $\mathrm{V}_{\mathrm{T} 2 \mathrm{~W}}$ and $\mathrm{V}_{\mathrm{ADC}}$ were less than $10.0 \mathrm{~cm}^{3}$. Five out of fifteen patients were found to have excellent agreement (DSC at least 0.70) between the target volumes delineated on T2W and ADC maps, and for these patients the $\mathrm{V}_{\mathrm{T} 2 \mathrm{~W}}$ and $\mathrm{V}_{\mathrm{ADC}}$ were at least $50.0 \mathrm{~cm}^{3}$. This was the case for Patient 1 (Fig. 1), where both target volumes delineated on ADC maps and T2W images were greater than $50.0 \mathrm{~cm}^{3}$, and the DSC was 0.75 . For the fifteen patients, we found that the average DSC between the ADC- and T2W-delineated target volumes was $0.54 \pm 0.22$.

Table 2 summarizes the percent edge contrast for the GTV delineated on ADC maps and T2W images for all fifteen patients. The edge contrast on ADC was found to be similar to or higher than that on T2W images for fourteen patients. An example of the difference in contrast between the delineated target volume and surrounding normal tissue is shown in Fig. 2 for Patient 3 with a 26.7\% edge contrast on ADC maps versus $10.4 \%$ on T2W images. For the fifteen patients, there was a statistically significant difference between the mean percentage edge contrast on ADC $(12.7 \pm 7.7 \%)$ versus that on $\mathrm{T} 2 \mathrm{~W}$ images $(4.6 \pm 3.2 \%$; $p=0.0010$ Sign test).

\section{Discussion}

Current practice for MR-guided intracavitary brachytherapy for cervix cancer is to define the GTV based on what is detected by clinical examination and visualized on MRI, typically T2W images. Here, we compared the use of DWI, namely ADC maps, against T2W imaging for delineation of the GTV. Using the percentage edge contrast as a quantifier, ADC maps provided significantly higher contrast near the periphery of delineated target volumes than T2W images. Using the DSC as a similarity measure, we observed disparities between the resulting $\mathrm{T} 2 \mathrm{~W}$ - and ADC-delineated target volumes. The worst agreement (DSC < 0.40) between GTVs delineated on ADC maps and $\mathrm{T} 2 \mathrm{~W}$ images was associated with $\mathrm{V}_{\mathrm{ADC}}$ and $\mathrm{V}_{\mathrm{T} 2 \mathrm{~W}}$ less than $10.0 \mathrm{~cm}^{3}$, with excellent agreement (DSC $\geq 0.70$ ) associated with $\mathrm{V}_{\mathrm{ADC}}$ and $\mathrm{V}_{\mathrm{T} 2 \mathrm{~W}}$ of at least $50.0 \mathrm{~cm}^{3}$. T2W-delineated volumes were generally larger than ADC-delineated volumes. As Figs. 3A-B demonstrate for Patient 9, it may be that the T2W images are leading to an overestimation of target volume size, since the contrast near the target periphery is not as high as that observed on ADC maps. It may also, or otherwise, be that the ADC maps are identifying only the most cellularly dense regions of the tumor, leading to smaller target volumes delineated on ADC maps (Fig. 3C). This may also explain why for another case, Patient 1 , there is excellent agreement on one pair of corresponding slices (Figs. 1A-B), but on another slice
Table 1. Comparison of GTV delineated on T2W images and $A D C$ maps (volumes reported in $\mathrm{cm}^{3}$ )

\begin{tabular}{lcccc} 
Patient & $\mathrm{V}_{\mathrm{T} 2 \mathrm{~W}-\mathrm{ADC}}$ & $\mathrm{V}_{\mathrm{T} 2 \mathrm{~W}}$ & $\mathrm{~V}_{\mathrm{ADC}}$ & $\mathrm{DSC}$ \\
\hline 1 & 52.1 & 76.1 & 62.5 & 0.75 \\
\hline 2 & 44.0 & 68.8 & 61.4 & 0.67 \\
\hline 3 & 21.1 & 43.7 & 24.6 & 0.62 \\
\hline 4 & 76.5 & 122.0 & 81.3 & 0.75 \\
\hline 5 & 11.2 & 21.3 & 19.5 & 0.55 \\
\hline 6 & 97.2 & 123.2 & 152.7 & 0.70 \\
\hline 7 & 0 & 2.7 & 0.4 & 0.00 \\
\hline 8 & 2.1 & 8.3 & 5.5 & 0.31 \\
\hline 9 & 14.7 & 28.8 & 23.5 & 0.56 \\
\hline 10 & 164.8 & 279.8 & 187.9 & 0.70 \\
\hline 11 & 33.1 & 49.2 & 68.7 & 0.56 \\
\hline 12 & 1.8 & 8.3 & 6.6 & 0.24 \\
\hline 13 & 11.6 & 39.9 & 15.9 & 0.42 \\
\hline 14 & 11.3 & 23.2 & 21.7 & 0.51 \\
\hline 15 & 44.6 & 65.0 & 52.5 & 0.76
\end{tabular}

the ADC map identifies selected regions inside what is delineated on the corresponding slice in the T2W image (Figs. 1C-D).

Although the mechanisms behind these discrepancies in GTV delineation are unclear at this point, it should be noted that similar findings have also been demonstrated between T2W MR images and fluoro-deoxy-glucose positron emission tomography/CT (FDG-PET/CT) images [17]. Ma et al. compared GTV contoured on T2W and on FDG-PET/CT images, and found that T2W images provided better visualization of larger target volumes than did FDG-PET/CT; however, FDG-PET/CT highlighted areas otherwise undetected on $\mathrm{T} 2 \mathrm{~W}$ images, especially for volumes less than $14.0 \mathrm{~cm}^{3}$ [17]. Thus, for this study it may be that ADC maps, like FDG-PET images, are highlighting areas undetected on the $\mathrm{T} 2 \mathrm{~W}$ images for smaller target volumes. Future studies should be performed comparing GTV delineation on T2W images to both ADC maps and FDG-PET/CT images. Furthermore, a range of target volumes should be included in the study to evaluate whether particular imaging modalities may be better suited to the identification of smaller target volumes.

Because histopathological confirmation of the delineated target volumes as tumors is not typically done for patients with intact cervix receiving brachytherapy, we were unable to assess the accuracy of the delineated volumes on ADC maps as part of this study. However, we refer to other studies which did use histopathological findings to assess the diagnostic accuracy of DWI and ADC values for cervix cancer [5, 6, 10, 11, 14]. Chen et al. found the sensitivity and specificity of DWI for detecting cervix cancer to be approximately $94-100 \%$ and $85 \%$, respectively [5]. With regard to ADC values, several investigators have shown a significantly lower median ADC value in malignant (ranging from 0.757 to $1.09 \times$ $10^{-3} \mathrm{~mm}^{2} / \mathrm{s}$ ) versus normal tissues (ranging from 1.33 to 

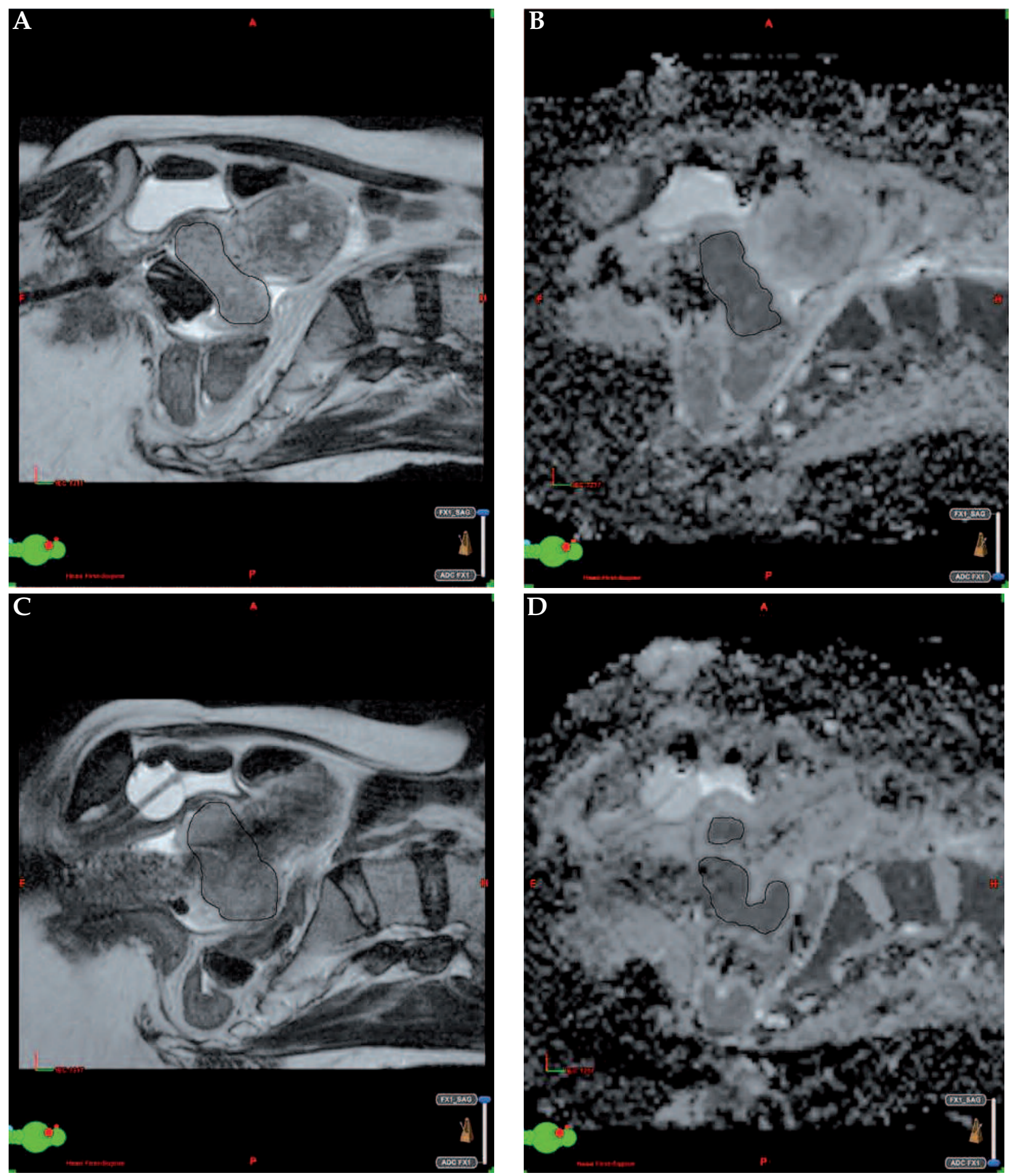

Fig. 1. The GTV delineated in black on T2W sagittal images $(A, C)$ and on the corresponding ADC sagittal maps (B,D) for Patient 1

$\left.2.09 \times 10^{-3} \mathrm{~mm}^{2} / \mathrm{s}\right)(p<0.001)[6,10,11,14]$. This difference in ADC values, when combined with the information from $\mathrm{T} 2 \mathrm{~W}$-images, improved the detection of cervix tumors [6]. Thus, these results support the assumption in this study that dark areas in the region of the cervix on ADC maps should represent malignant tissues. Future studies should be performed on both ADC maps and T2W images to assess the accuracy of the delineated target volumes as tumor using patient populations that will facilitate histopathological confirmation.

Lastly, it should also be noted that a particular weakness of DWI is that it is highly susceptible to image distortions caused by the presence of metal. Thus, for brachytherapy planning using metal applicators, T2W images should remain the primary image dataset for defining the GTV, and it is recommended that DWI be used as 
a supplement to, but not in place of, T2W imaging. Because the disagreement in GTV between ADC maps and T2W images observed here may also be due to the presence of greater distortions in DWI versus T2W images, future studies should be performed comparing DWI to T2W imaging for cervix target delineation in patients with no brachytherapy applicator in place.

\section{Conclusions}

Previous work has demonstrated that ADC values are lower in tumors than in normal tissues for cervix cancer [6-13]. Here, we investigate how this previously reported difference in ADC value between malignant and normal cervix tissues affects target visibility and target volume delineation on ADC maps, as compared to the current standard of T2W images. ADC maps derived from DWI were found to display superior definition of the target volume edge relative to $\mathrm{T} 2 \mathrm{~W}$ images. ADC-delineated gross target volumes were also found to be generally smaller than T2W-delineated target volumes. These results suggest that incorporating the use of DWI for image-guided radiotherapy may yield GTVs that are different from those based on $\mathrm{T} 2 \mathrm{~W}$ images alone.

This is the first study to investigate the clinical application of this difference in ADC value to the delineation of GTV on ADC maps for image-guided intracavitary brachytherapy. Future studies are still needed to investigate other aspects of DWI and ADC maps for cervix cancer radiotherapy, e.g., how these modalities compare to FDG-PET imaging, how accurate these modalities are for tumor delineation, and how sensitive these modalities are to the presence of metal applicators.

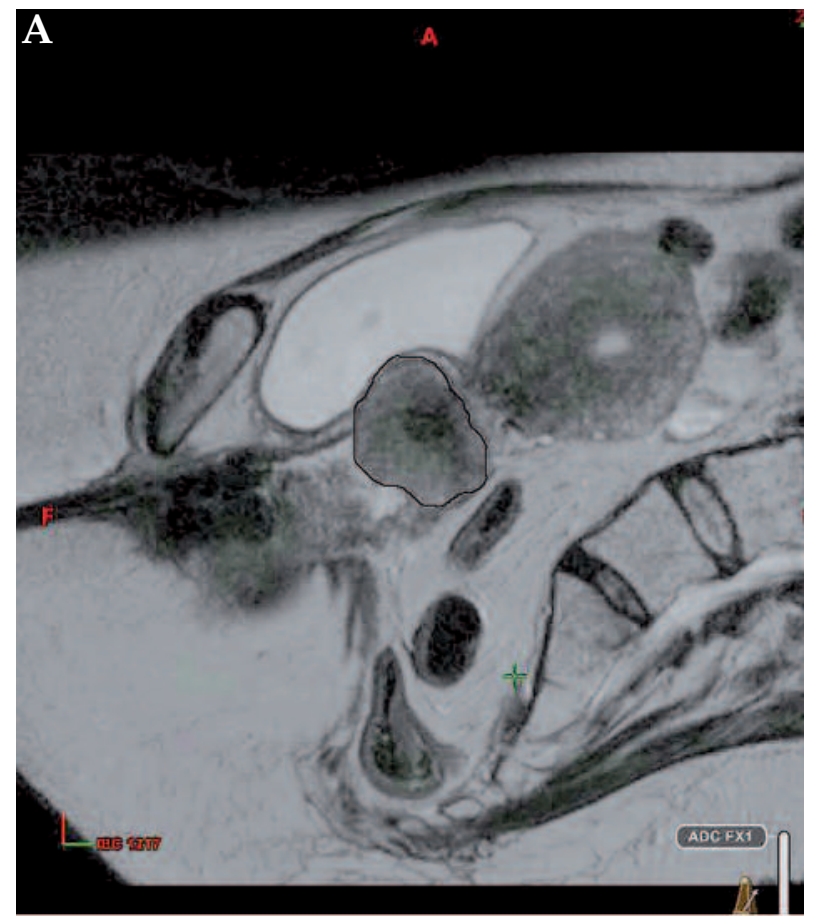

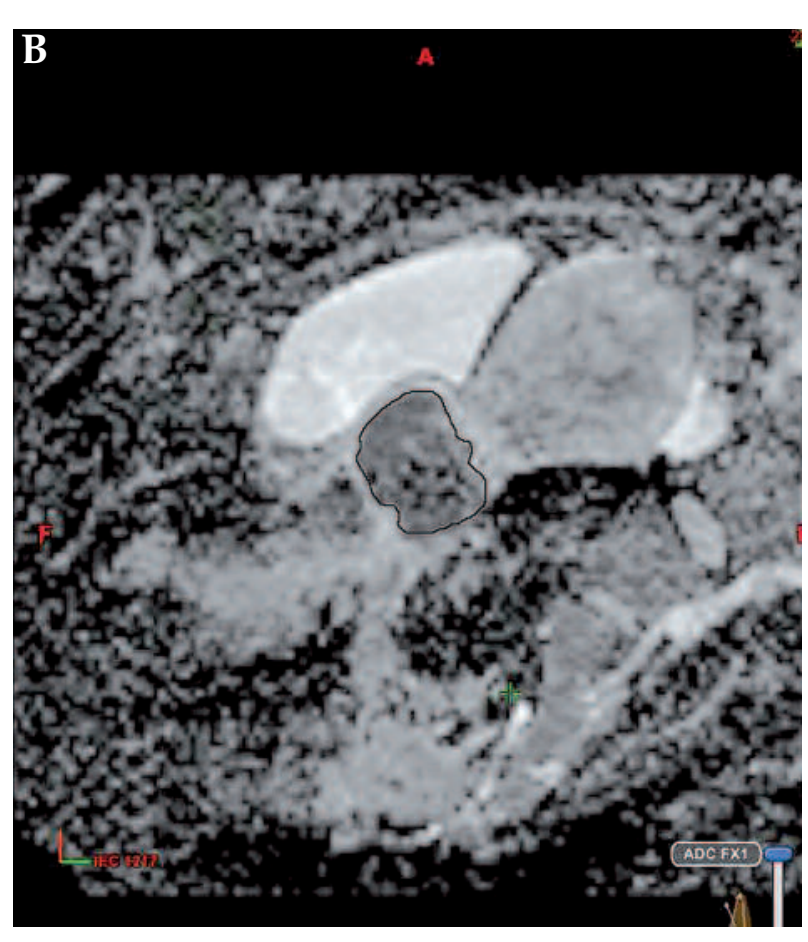

Table 2. Comparison of tumor edge visibility on $\mathrm{T} 2 \mathrm{~W}$ images and $\mathrm{ADC}$ maps

\begin{tabular}{lcc} 
Patient & \% Edge contrast on T2W & \% Edge contrast on ADC \\
\hline 1 & 4.2 & 28.7 \\
\hline 2 & 5.3 & 14.0 \\
\hline 3 & 10.4 & 26.7 \\
\hline 4 & 8.1 & 20.5 \\
\hline 5 & 3.9 & 9.6 \\
\hline 6 & 1.1 & 9.3 \\
\hline 7 & 0.4 & 11.7 \\
\hline 8 & 7.2 & 3.8 \\
\hline 9 & 9.8 & 10.5 \\
\hline 10 & 3.2 & 16.7 \\
\hline 11 & 2.7 & 13.8 \\
\hline 12 & 2.0 & 2.9 \\
\hline 13 & 6.0 & 6.2 \\
\hline 14 & 0.7 & 10.5 \\
\hline 15 & 4.0 & 6.2 \\
\hline
\end{tabular}

\section{Acknowledgments}

We would like to acknowledge Jian Ming-Zhu, Ph.D. for his assistance in the initial design of the MR image acquisition protocols. We would like to thank Stacie Mackey, RT(R) for her continued support of the analysis and acquisition of the MR images. We would like to thank Todd A. DeWees, PhD, for his assistance with the statistical analyses. This work was supported in part by 1R01CA136931.

Fig. 2. The GTV delineated in black on a T2W sagittal image (A) and on the corresponding ADC sagittal map (B) for Patient 3 

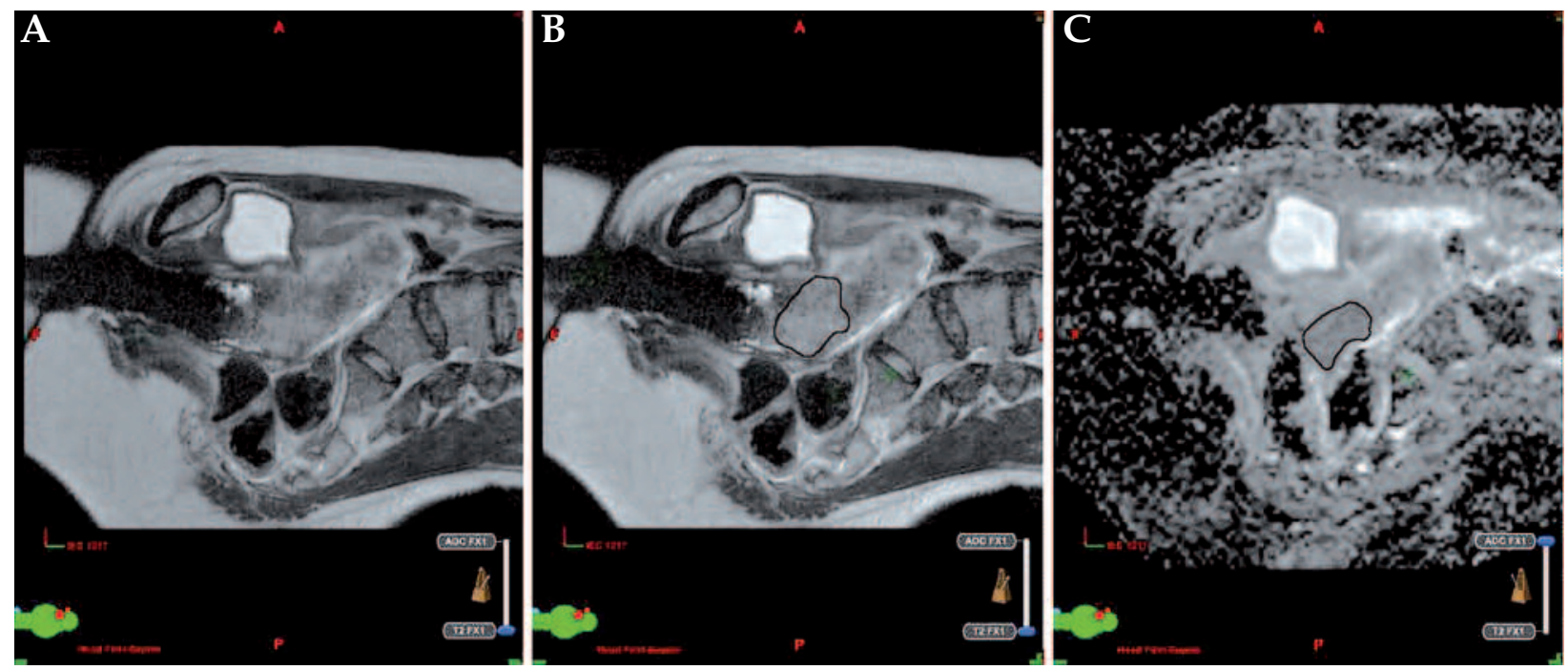

Fig. 3. A T2W sagittal image showing cervix tumor with fairly indistinct borders (A), the GTV delineated in black on the same T2W sagittal image (B) and on the corresponding ADC sagittal map (C) for Patient 9

\section{Disclosure}

The authors have no conflicts of interest to disclose with regard to this manuscript.

\section{References}

1. Mitchell DG, Snyder B, Coakley F et al. Early invasive cervical cancer: tumor delineation by magnetic resonance imaging, computed tomography, and clinical examination, verified by pathologic results, in the ACRIN 6651/GOG 183 Intergroup Study. J Clin Oncol 2006; 24: 5687-5694.

2. Haie-Meder C, Potter R, Van Limbergen E et al. Recommendations from Gynaecological (GYN) GEC-ESTRO Working Group (I): concepts and terms in 3D image based 3D treatment planning in cervix cancer brachytherapy with emphasis on MRI assessment of GTV and CTV. Radiother Oncol 2005; 74: 235-245.

3. Nag S, Cardenes H, Chang S et al. Proposed guidelines for image-based intracavitary brachytherapy for cervical carcinoma: report from Image-Guided Brachytherapy Working Group. Int J Radiat Oncol Biol Phys 2004; 60: 1160-1172.

4. Potter R, Haie-Meder C, Van Limbergen E et al. Recommendations from gynaecological (GYN) GEC ESTRO working group (II): concepts and terms in 3D image-based treatment planning in cervix cancer brachytherapy-3D dose volume parameters and aspects of 3D image-based anatomy, radiation physics, radiobiology. Radiother Oncol 2006; 78: 67-77.

5. Chen J, Zhang Y, Liang B et al. The utility of diffusion-weighted MR imaging in cervical cancer. Eur J Radiol 2009; 74 . 101-106.

6. Charles-Edwards EM, Messiou C, Morgan VA et al. Diffusionweighted imaging in cervical cancer with an endovaginal technique: potential value for improving tumor detection in stage Ia and Ib1 disease. Radiology 2008; 249: 541-550.

7. Harry VN. Novel imaging techniques as response biomarkers in cervical cancer. Gynecol Oncol 2010; 116: 253-261.

8. Kilickesmez O, Bayramoglu S, Inci E et al. Quantitative diffusion-weighted magnetic resonance imaging of normal and diseased uterine zones. Acta Radiol 2009; 50: 340-347.

9. Liu Y, Bai R, Sun H et al. Diffusion-weighted magnetic resonance imaging of uterine cervical cancer. J Comput Assist Tomogr 2009; 33: 858-862.
10. McVeigh PZ, Syed AM, Milosevic M et al. Diffusion-weighted MRI in cervical cancer. Eur Radiol 2008; 18: 1058-1064.

11. Naganawa S, Sato C, Kumada H et al. Apparent diffusion coefficient in cervical cancer of the uterus: comparison with the normal uterine cervix. Eur Radiol 2005; 15: 71-78.

12. Payne GS, Schmidt M, Morgan VA et al. Evaluation of magnetic resonance diffusion and spectroscopy measurements as predictive biomarkers in stage 1 cervical cancer. Gynecol Oncol 2010; 116: 246-252.

13. Xue HD, Li S, Sun F, Sun HY et al. Clinical application of body diffusion weighted MR imaging in the diagnosis and preoperative N staging of cervical cancer. Chin Med Sci J 2008; 23: 133-137.

14. Levy A, Medjhoul A, Caramella C et al. Interest of diffusionweighted echo-planar MR imaging and apparent diffusion coefficient mapping in gynecological malignancies: a review. J Magn Reson Imaging 2011; 33: 1020-1027.

15. Rolhlfing R, Brandt R, Menzel R et al. Quo vadis, atlas-based segementation? In: The Handbook of Medical Image Analysis. Volume III: Registration Models. Suri J, Wilson DL, Laxminarayan S (eds.). Kluwer Academic/Plenum Publishers, New York; Chapter ii: $435-486$.

16. Zijdenbos AP, Dawant BM, Margolin RA et al. Morphometric analysis of white matter lesions in MR images: method and validation. IEEE Trans Med Imaging 1994; 13: 716-724.

17. Ma DJ, Zhu J, Grigsby PW. Utility of FDG-PET in Defining a High Risk GTV for Cervix Cancer. Int I Radiat Oncol Biol Phys 2009; 75: S148-149. 